\title{
Delirium management by Palliative Medicine specialists: a survey from the Association for Palliative Medicine of Great Britain and Ireland
}

Short title: Delirium management by palliative medicine specialists

Jason W Boland ${ }^{1 *}$, Monisha Kabir ${ }^{2 *}$, Shirley H Bush ${ }^{2,3}$, Juliet Spiller ${ }^{4}$, Miriam J Johnson ${ }^{1}$, Meera Agar ${ }^{5}$, Peter G Lawlor ${ }^{2,3}$

${ }^{1}$ Wolfson Palliative Care Research Centre, Hull York Medical School, University of Hull, Hull, UK

${ }^{2}$ Bruyère Research Institute; Bruyère Continuing Care; Ottawa Hospital Research Institute, Ottawa, Ontario, Canada

${ }^{3}$ Department of Medicine, Division of Palliative Care, University of Ottawa

${ }^{4}$ Marie Curie Hospice, Edinburgh, UK

${ }^{5}$ IMPACCT (Improving Palliative, Chronic and Aged Care through Clinical Research and Translation), Faculty of Health, University of Technology Sydney

* Joint first authors (JWB and MK contributed equally to this paper).

Corresponding author: Jason W Boland, Wolfson Centre for Palliative Care Research, Hull York Medical School, University of Hull, Hull, UK. Email: Jason.Boland@hyms.ac.uk

Word count: 3425

(C) Author(s) (or their employer(s)) 2019. No commercial re-use. 


\begin{abstract}
Objectives: Delirium is common in palliative care settings. Management includes detection, treatment of cause(s), non-pharmacological interventions and family support; strategies which are supported with varying levels of evidence. Emerging evidence suggests that antipsychotic use should be minimised in managing mild to moderate severity delirium, but the integration of this evidence into clinical practice is unknown.

Methods: A 21-question online anonymous survey was emailed to Association for Palliative Medicine members in current clinical practice $(n=859)$, asking about delirium assessment, management and research priorities.

Results: Response rate was 39\%: $70 \%$ of respondents were palliative medicine consultants. Delirium guidelines were used by some: $42 \%$ used local guidelines but $38 \%$ used none. On inpatient admission, $59 \%$ never use a delirium screening tool. Respondents would use non-pharmacological interventions to manage delirium, either alone (39\%) or with an antipsychotic (58\%). Most respondents $(91 \%)$ would prescribe an antipsychotic, and $6 \%$ a benzodiazepine, for distressing hallucinations unresponsive to nonpharmacological measures. Inpatient $(57 \%)$ and community teams $(60 \%)$ do not formally support family carers. Research priorities were delirium prevention, management and prediction of reversibility.

Conclusion: This survey of UK and Irish Palliative Medicine specialists shows that delirium screening at inpatient admission is suboptimal. Most specialists continue to use antipsychotics in combination with non-pharmacological interventions to manage delirium. More support for family carers should be routinely provided by clinical teams. Further rigorously designed clinical trials are urgently needed in view of management variability, emerging evidence and perceived priorities for research.
\end{abstract}

Keywords: Palliative Medicine, Palliative Care, Delirium Management, Cognition, Assessment, Research 


\section{INTRODUCTION}

Delirium is an acute onset neurocognitive disorder characterised by disturbance in attention, awareness and cognition, which fluctuates during a 24-hour period.[1] Delirium has multiple aetiologies and is common in patients receiving palliative care. $[2,3]$ It is present in $13-42 \%$ of patients admitted to specialist palliative care inpatient units, 26-62\% during admission, and 59-88\% in the last weeks of life.[4] It is associated with falls,[5] increased cognitive impairment after discharge,[6] and a poor prognosis.[3,7] Delirium causes high levels of distress for patients, their families and healthcare practitioners.[8-10]

Delirium is underdiagnosed across many clinical settings including cancer and palliative care.[11-14] In one study of palliative care inpatient consults, delirium diagnosed subsequently by a palliative medicine specialist had been missed by the primary referring team in $61 \%$ of patients.[11] Patients with missed delirium were frequently referred for pain control.[11] In people with advanced disease, dementia or generalized central nervous system dysfunction, the diagnosis of delirium poses challenges.[15] Furthermore, hypoactive delirium, the most underdiagnosed subtype of delirium, is the most prevalent subtype (68-86\% of cases) in palliative care settings.[4]

Detection and assessment/diagnosis is the first critical step; failure to conduct an adequate assessment commonly results in a missed diagnosis.[2, 11] Delirium assessment based solely on clinical judgement leads to under-detection. $[2,11]$ Validated assessment tools exist for screening, diagnosing and monitoring the severity of delirium in cancer and palliative care populations.[2,11]

The management of delirium is multifaceted and multidisciplinary. It includes assessment, treating the underlying causes if appropriate (50\% of episodes are reversible), non-pharmacological interventions and family support.[2, 11, 16, 17] Family caregivers are important in the management of a patient with delirium.[8-10] Multicomponent interventions, integrating volunteers and family members, have demonstrated major reductions in delirium incidence and length of delirium cases in older ( $\geq 70$ years of age) hospitalized patients compared to control groups receiving usual care.[18]. Pharmacological management is commonly used to specifically treat the symptoms of delirium or provide sedation for the agitated patient. Although not specifically targeting populations receiving palliative care, recent systematic reviews in heterogeneous populations do not support antipsychotic use in hospitalized patients with delirium.[19, 20]

There are varied levels of evidence for the management of delirium and its distressing clinical components, and not all have been evaluated specifically in palliative care. As such, clinician opinion drives practice in this setting. Based largely on a double-blind, non-placebo-controlled trial,[21] haloperidol has been the "practice standard" in the symptomatic management of delirium in palliative care.[22] In the largest randomized controlled trial (RCT) of palliative care patients $(\mathrm{N}=247)$ with mild to moderate severity delirium (assessed by the Memorial Delirium Assessment Scale (MDAS)) and with Australia-modified Karnofsky Performance Status of 30-50\%, the severity and duration of behavioural, communication and perceptual symptoms of delirium were greater in those who received either risperidone or haloperidol compared to placebo.[23] In a recent single-centre RCT, hospitalized advanced cancer patients with persistent agitated delirium, Karnofsky Performance status of 10-40\% treated with haloperidol, additional lorazepam increased the level of sedation at 8 hours compared to placebo.[24] Neither lorazepam nor placebo improved the overall severity of delirium. The trial did not include a haloperidol placebo arm and does not inform the debate regarding the place of antipsychotics in the management of delirium. A Cochrane review found "insufficient evidence" for drug therapy specific to delirium in terminally ill adult patients, suggesting that further research is necessary and that health care professionals should remain in line with up-to-date clinical guidelines.[25] 
In view of this emerging evidence, there is a need to determine the current clinical practice of specialist palliative medicine physicians regarding their approach to delirium assessment, management and research prioritisation.

\section{METHODS}

Study design

A cross-sectional online survey design to collect quantitative and qualitative data.

Survey development

A review of the literature and previously published surveys informed the development of this online survey. The draft survey was developed and reviewed by experts in palliative medicine. After pilot testing among Marie Curie Palliative Medicine specialists in the UK $(N=24)$ it was further modified based on their feedback (Table 1).

Table 1. Outline of survey contents (see Appendix 1 for full survey)

\begin{tabular}{ll}
\hline Sections & Key information \\
\hline Section I (Questions 1-5): & $\bullet$ Current position/role \\
Demographic details & $\bullet$ Number of years in practice \\
& $\bullet$ Practice settings of respondent (more than one response possible \\
& if applicable) \\
& - Delirium screening practices and tools used, if any
\end{tabular}

Section II (Questions 6-10): Cognitive and delirium screening as part of inpatient care

Section III (Questions 11-13): Delirium in the home setting and referral for inpatient admission in the last year

Section IV (Questions 14-17): Specific delirium practices as part of inpatient care

Section V(Questions 18, 19): Education and support of family carers
- Delirium screening tools used, if any

- Frequency of screening tool use

- Number of patients seen with delirium in the community in the preceding year and the need for inpatient admission care for these patients

- Factors influencing decision to recommend inpatient admission

- Use of guidelines in symptomatic treatment of delirium

- Use of non-pharmacological interventions in delirium management

- *Clinical scenario and respondents' choice of pharmacological management

- Proportion of patients for which deep or palliative sedation was recommended at end of life

- Formal support mechanisms in respondents' practice setting(s) for family members of patients with delirium (more than one response possible if applicable) 
Section VI (Question 20):

Delirium research priorities in

palliative care

Survey conclusion (Question 21): *NB. The clinical scenario posed a patient with distressing hallucinations due to delirium unresponsive to
non-pharmacological measures.

\section{Survey sampling}

The Association for Palliative Medicine (APM) of Great Britain and Ireland is the largest organization of specialist Palliative Medicine physicians in the UK/Ireland, with over 1000 members (https://apmonline.org/). Eligibility criteria restricted the survey to currently practicing Palliative Medicine specialists. These physicians see patients with life-limiting conditions, including cancer (any site) and non-cancer illnesses.

\section{Survey and procedure}

The survey was administered online via a secure online platform, SurveyMonkey ${ }^{\circledR}$ (www.surveymonkey.com/). The final 21-question anonymous survey weblink was emailed by the APM Secretariat to preserve anonymity to all members of the APM $(N=1121 ; 859$ of which were currently practicing Palliative Medicine specialists according to APM records) on February 28, 2017. Three followup reminder emails were sent out to non-responders. The survey could only be completed once by each participant. Skip logic facilitated answers to specific practice setting-related questions.

\section{Data analysis}

Descriptive statistics, percentages, and weighted averages were computed for the appropriate quantitative data study variables using Microsoft Excel $^{\circledR}$ (2010). Surveys of respondents who did not identify themselves as Palliative Medicine specialists in current practice, or who had missing data for the first five demographic questions were excluded from analysis.

\section{Ethics}

Hull York Medical School Research Ethics approval was attained prior to survey distribution.

\section{RESULTS}

Of 1121 APM members surveyed between February 28, 2017 and July 22, 2017, 364 responded. Out of 364 responses, 32 were excluded due to not being in current practice, not identifying themselves as Palliative Medicine specialists or not completing the survey's first five demographic questions. Of the 859 APM members who were known Palliative Medicine specialists in current practice, the overall survey response rate was 39\% (332/859); (Table 2). Not all respondents answered all questions, so the denominator is variable for subsequent questions.

Table 2. Demographics of the 332 Palliative Medicine specialist respondents (Survey questions 1-5)

\begin{tabular}{lll}
\hline Characteristics & Subcategories & $\boldsymbol{N}(\boldsymbol{\%})^{\dagger}$ \\
\hline Role $^{\$}$ & Consultant in Palliative Medicine & $232(70)$
\end{tabular}


Staff grade or Associate Specialist in Palliative Medicine

Specialist Trainee or Registrar in Palliative

Medicine
$<1$ year

$1-4$ years

$5-10$ years

$>10$ years

Only inpatient care

Only community care

Both inpatient and community care

Cancer Centre

Community Hospital

General Hospital

Hospice Care

Long Term Care / Nursing Home

Patient's Home

Teaching Hospital

Other
$50(15)$

$50(15)$

81 (24)

202 (61)

$130(39)$

9 (3)

$193(58)$

$62(19)$

45 (14)

146 (44)

265 (80)

63 (19)

$169(51)$

119 (36)

9 (3)

$0(0)$

12 (4)

$83(25)$

111 (33)

$126(38)$

${ }^{\top}$ Total $N=332$

* If participants worked in multiple settings, they were able to select all that applied.

$\$$ Consultant: fully trained specialist in Palliative Medicine; Staff grade or Associate Specialist in Palliative Medicine: non-consultant grade specialist in Palliative Medicine; Specialist Trainee or Registrar in Palliative Medicine: physician undergoing Palliative Medicine speciality training.

\section{Screening tool use}

In the questions regarding tool and guideline use, respondents were asked to answer on behalf of the palliative care team within which they worked. Table 3 summarises screening tool use for cognitive deficits/dementia and delirium.

Respondents who indicated that their palliative care teams use a tool to screen for cognitive deficits or dementia at inpatient assessment commonly used the Mini-Mental State Examination (MMSE) (143/231; $62 \%$ ), with the 4'A's Test (4AT) and Montreal Cognitive Assessment (MoCA) each being used by $10 \%$ (24/231). The Short Orientation-Memory-Concentration Test (SoMCT)/Blessed Orientation-MemoryConcentration (BOMC) $(10 / 231 ; 4 \%)$ and Addenbrooke's Cognitive Examination (ACE-R) $(2 / 231 ; 1 \%)$ were less frequently used to screen for cognitive deficits or dementia at inpatient assessment. Other tools, such as the CAM, AMT, clock drawing task and six-item Cognitive Impairment Test (6CIT), were used by $12 \%(28 / 231)$ of teams. 
Respondents who indicated that their palliative care teams use a tool to screen for delirium on the first inpatient assessment regularly used the Confusion Assessment Method (CAM) (59/127; 46\%), followed by the 4AT (28/127; 22\%). Other tools, such as The Delirium Observation Screening Scale (DOS), Family Confusion Assessment Method (FAM-CAM), Single Question in Delirium (SQiD), MMSE, the MoCA and Abbreviated Mental Test (AMT) were used by 32\% (40/127).

Table 3. Descriptive results of screening tool use

\begin{tabular}{|c|c|c|c|c|c|c|c|c|}
\hline & Question stem & Total $N$ & $\begin{array}{l}\text { Never } \\
N(\%)\end{array}$ & $\begin{array}{l}\text { Sometimes } \\
N(\%)\end{array}$ & $\begin{array}{l}\text { Often } \\
N(\%)\end{array}$ & $\begin{array}{l}\text { Very } \\
\text { Often } \\
N(\%)\end{array}$ & $\mathbf{S D}^{\dagger}$ & $\begin{array}{l}\text { Weighted } \\
\text { average* }\end{array}$ \\
\hline Q6 & $\begin{array}{l}\text { My palliative care } \\
\text { team uses a tool to } \\
\text { screen for cognitive } \\
\text { deficits or dementia at } \\
\text { inpatient assessment. }\end{array}$ & 321 & $87(27)$ & $169(53)$ & $26(8)$ & 39 (12) & 32.4 & 2.1 \\
\hline Q8 & $\begin{array}{l}\text { My palliative care } \\
\text { team uses a tool to } \\
\text { screen for delirium on } \\
\text { the first inpatient } \\
\text { assessment. }\end{array}$ & 314 & $184(59)$ & $88(28)$ & $14(4)$ & $28(9)$ & 38.7 & 1.6 \\
\hline Q10 & $\begin{array}{l}\text { My palliative care } \\
\text { team uses a delirium } \\
\text { screening tool on an } \\
\text { ongoing basis for } \\
\text { inpatients. }\end{array}$ & 125 & $32(26)$ & $82(66)$ & $6(5)$ & $5(4)$ & 36.1 & 1.9 \\
\hline
\end{tabular}

Delirium guideline use

Delirium guidelines were inconsistently used, with $42 \%(115 / 276)$ of specialist palliative care teams using local guidelines and 38\% (104/276) using none. Others $(57 / 276 ; 21 \%)$ stated they used other guidelines such as The National Institute for Health and Care Excellence (NICE), Scottish Intercollegiate Guidelines Network (SIGN), Palliative Adult Network Guidelines, and Palliative Care Formulary.

\section{Delirium care in the community}

The majority $(135 / 176 ; 77 \%)$ of Palliative Medicine specialists would recommend inpatient admission to either hospital or hospice in no more than $50 \%$ of patients seen with delirium in their own homes over the past year. Reasons for admission in the context of delirium varied but was most commonly due to inability of family caregivers to cope (Figure 1).

\section{Non-pharmacological interventions}

Most respondents would routinely use non-pharmacological interventions (such as pursue optimal sound and/or lighting levels; gently re-orientate; facilitate the presence of a family member) to manage delirium, either alone $(106 / 275 ; 39 \%)$ or more commonly with an antipsychotic $(160 / 275 ; 58 \%)$. Nine out of 275 $(3 \%)$ would not routinely use non-pharmacological interventions. 


\section{Sedation}

Out of 271 respondents, $130(48 \%)$ reported rarely ( $<5 \%$ of cases) prescribing or recommending deep or palliative sedation for inpatients in the last days/weeks of life with non-reversible, refractory agitated delirium. Twenty-seven percent (73/271) reported doing so in 5-20\% of cases, with $10 \%$ (28/271) in 21 $40 \%$ of cases, $8 \%(23 / 271)$ in $41-60 \%$ of cases, and $6 \%$ (17/271) of respondents doing so in most (>60\%) of the patients they see in this situation.

Respondents commented on their perspectives on sedation in the final open-ended question, indicating it was hard to distinguish between alleviating distress and providing palliative sedation) and open to a wide range of interpretations.

\section{Clinical scenario}

Most physicians would prescribe an antipsychotic as an initial medication choice 248/273 (91\%), with 190/273 (70\%) using typical antipsychotics and 43/273 (16\%) using atypical antipsychotics first-line. Six percent (16/273) would prescribe a benzodiazepine, 5\% (15/273) would prescribe combination antipsychotic and benzodiazepine and 2\% (5/273) would not use medication. In open-ended responses, respondents indicated uncertainty about using antipsychotics for the management of delirium after the publication of the Agar et al. RCT.[23] Respondents also indicated the importance of addressing reversible causes of delirium on a case-by-case basis and the challenges in optimising nonpharmacological interventions in busy inpatient settings.

\section{Support of family carers}

Most inpatient $(153 / 270 ; 57 \%)$ and community teams $(97 / 161 ; 60 \%)$ do not formally support family carers in a systematic or standardized manner. Palliative care specific information leaflets about delirium were only provided as a standard approach in 10\% (27/270) of teams in inpatient settings and $7 \%$ in the community. Similarly, a generic information leaflet about delirium was not widely provided as part of inpatient $(22 / 270 ; 24 \%)$ and community services $(10 / 161 ; 6 \%)$. Informal verbal support was offered by $24 \%(64 / 270)$ in the inpatient setting and 22\% (35/161) in community settings.

\section{Delirium research priorities}

Research priorities were prevention, prediction of reversibility and management (non-pharmacological and pharmacological). Respondents $(\mathrm{n}=270 / 335 ; 81 \%)$ rated the importance $(0=$ not at all, and $4=$ extremely important, as anchors) of domains for future research; the three highest weighted average scores were for research on preventive strategies (2.91/4), prediction of delirium reversibility (2.96/4), and the pharmacological and non-pharmacological management of delirium (3.19/4).

\section{DISCUSSION}

This survey represents many experienced UK and Irish palliative medicine physicians. Over a third never used delirium clinical guidelines, although just under half used local guidelines. Most never used delirium screening tools. Just over half use non-pharmacological interventions to manage delirium. For distressing hallucinations unresponsive to non-pharmacological measures, most prescribe antipsychotics, but very few a benzodiazepine. Family support was informal. Delirium was reported a clinically important topic with uncertainties about best practice. Research priorities were delirium prevention, management and 
prediction of reversibility.

\section{Tool and guideline use}

Screening tools are used only if delirium is clinically suspected, rather than systematically on admission and during the patient's care. This is at variance with the literature and guideline recommendations for delirium screening, which advise screening for delirium using a tool, and patient/family consultation on admission and on an ongoing basis to assess fluctuations in risk factors causing delirium and in order to detect delirium early.[26, 27]

Delirium guidelines were not used regularly by most of the teams, and when used, were mostly local guidelines. Although the NICE guidelines have a national UK purview, they are not specific to palliative care and excluded people at the end of life from their recommendations.[27]. This identifies a major issue with delirium guidelines; although patients with advanced illness are cared for in the settings for which these guidelines apply, there is little about how the evidence relating to caring for someone with delirium applies to patients at the end of life. There are other delirium guidelines available for national use, such as the Canadian Coalition for Seniors' Mental Health (CCSMH) guidelines, which are specific for older people at the end of life, although these are infrequently used according to this study's findings, suggesting a lack of geographical reach.

Our results contrast with a survey of European Delirium Association (EDA) members (only $4 \%$ were palliative care specialists), of whom 52\% always or mostly use a scale to assess delirium, typically either the CAM (52\%) or DOSS (30\%), and 86\% would review at least once a day.[28] Most respondents (78\%) reported that their institutions had specific guidelines for delirium.[28]

Similar to cognitive screening, delirium screening tools were seldom used, and rarely used consistently. When used, the CAM and 4AT were most commonly used. These results are consistent with guidelines, NICE advises use of the short CAM or Diagnostic and Statistical Manual of Mental Disorders (DSM) IV for diagnosis [27] and CCSMH recommends use of the CAM as a diagnostic measure in conjunction with the MMSE for assessment of cognition.[29] A survey of Palliative Medicine specialists found the MMSE (39\%), AMTS (27\%) or clinical judgement alone (35\%) was used to identify cognitive impairment.[30]

In this survey, the MMSE was used by the majority of respondents to screen for cognitive deficits or dementia, whereas a few respondents used the MMSE to screen for delirium at inpatient assessment. The MMSE is neither designed nor recommended as a diagnostic tool to confirm delirium.[2] Thus, there may be a need for improved clinical uptake of delirium screening tools as an initial screening method, although some palliative care patients find it difficult to complete, so a shorter cognitive test could be used.[2, 17, 26]

\section{Delirium care in the community}

Most patients with delirium were deemed manageable at home. These findings are echoed in an Australian survey, where palliative medicine specialists would generally manage delirium in the patients' home.[31]

In this survey, the inability of family caregivers to cope was the most common reason for admission. As a change in environment can increase problems for older people with cognitive issues and delirium,[27] if carer stress and coping can be supported and the patient can be managed at home, care at home might be warranted. Providing support and education for family caregivers is not only essential in the management of delirium but is also important in potentially reducing admissions and family distress. 


\section{Non-pharmacological interventions}

Although most respondents indicated that they use non-pharmacological strategies to manage delirium either in conjunction with an antipsychotic (58\%) or as a sole strategy (39\%), this should be an essential part of care by all clinical teams.[16, 26] In an Australian survey, $85 \%$ of palliative medicine specialists would generally use non-pharmacological management.[31] In an European Delirium Association survey, $67 \%$ initially used non-pharmacological management in hypoactive delirium and $61 \%$ initially used a combination of non-pharmacological and pharmacological interventions for hyperactive delirium.[28]

\section{Sedation}

Palliative sedation has been defined as administration of sedative drugs to reduce the consciousness of a terminal patient as much as necessary to relieve refractory symptoms.[32] "Deep or palliative sedation" was used in the survey question stem and some respondents reported being unable to accurately answer the question. The overwhelming response was that sedation might be an unintended consequence of pharmacologically managing the delirium. Palliative sedation was rarely used by respondents, accepting that patients might become sedated as a result of a balanced approach to controlling symptoms and delirium, though sedation was not the primary intention of therapy. Although this is in line with UK practice,[33] a systematic review identified refractory agitation as the main symptomatic indication for palliative sedation.[34]

\section{Case scenario of management of a patient with agitated delirium}

Most respondents would prescribe an antipsychotic for a patient with distressing hallucinations, delusions and agitation unresponsive to non-pharmacological measures. The role of antipsychotics in the management of distressing delirium with severe specific symptoms in patients receiving palliative care is currently uncertain, $[23,24,35]$ with prior placebo-controlled data limited to patients with HIV,[21] until more recently with current available placebo-controlled data exploring specific clinical scenarios.[23, 24] In the two most recent trials one included participants with mild to moderate delirium, who were experiencing specific target symptoms;[23] and the other included participants at the end of their life who remained agitated despite regular haloperidol.[24] Both trials allowed optimal treatment of reversible precipitants and non-pharmacological measures, as clinically determined by the multidisciplinary team; and asked the pragmatic question of the role of medications for shorter term symptomatic management in addition to these other established approaches of clinical care.[23, 24, 35] Both had placebo arms and this provides data on impact on symptom resolution of the individualised optimal treatment of reversible precipitants and non-pharmacological measures, acknowledging this includes multifactorial measures which will vary between participants.[23, 24]

Benzodiazepines were indicated as being rarely used for this scenario, while a similar UK survey found that $34.5 \%$ (59/171) would prescribe benzodiazepines on an as needed basis.[30] The aforementioned lorazepam vs. placebo trial (single dose when added to regular haloperidol for a single episode of agitation or restlessness) in participants who remained agitated despite regular haloperidol, showed that whilst sedation was deeper for the lorazepam group at 8 hours, those in the placebo group improved to a Richmond Agitation-Sedation Scale score of around zero (alert and calm) which would appear to be the more optimal goal, especially for those who had potentially reversible delirium.[24]

In the RCT by Agar et al. (published 3-months before this survey was initially sent out), patients received treatment of the cause of delirium and supportive strategies. However, patients receiving risperidone or haloperidol had higher delirium symptom scores compared to placebo. The majority of participants had mild-moderate overall delirium severity and average doses of administered haloperidol were low.[23]. In 
an Australian survey, $62 \%$ of palliative medicine specialists stated antipsychotics were the pharmacological "agent of choice" for symptomatic delirium management; for $38 \%$ it was benzodiazepines.[31]. In an audit of patients $\geq 65$ years admitted to hospital with delirium, antipsychotics were used in $66 \%$ patients with severe behavioural and or emotional disturbance associated with delirium, but in only $17 \%$ if these symptoms were not severe.[36] In contrast, benzodiazepines were used in $38 \%$ with severe symptoms and 18\% without.[36] However, these two studies predated the Agar et al. RCT.[23] Some respondents reported that the study by Agar et al. [23] has made them aware of the poor evidence base for antipsychotics in this setting. Some clinicians report difficulty in applying these findings to their clinical practice and/or don't feel that current study findings fully represent their clinical practice, and, as the study's authors state, does not inform practice for people with severe delirium;[23] and hence many report continuing use in their clinical practice.

\section{Support of family carers}

As most teams do not formally support family carers of patients with delirium, this is an essential area for improvement for both community and inpatient teams. Informal verbal support supported by information leaflets, preferably specific to palliative care, (such as the delirium information leaflet accessible from: https://www.bruyere.org/en/Delirium) may allow for improved formal support of family caregivers.

The literature indicates a need for formal support for family members, demonstrated when families of hospitalised older relatives with delirium described the experience as distressing.[10] Studies highlight the necessity of informational skills and resources needed to mitigate family distress and provide support, including information which addresses family concerns.[8, 37] Although more data is needed, family caregiver education may be an important intervention to improve outcomes for both patient and caregiver.[9]

\section{Research priorities}

All proposed areas of research were important to respondents, with preventive strategies, prediction of delirium reversibility, and the pharmacological and non-pharmacological management of delirium being the most important. Further methodologically rigorous studies in palliative care populations using standardized outcome measures are needed.

\section{Limitations}

A response rate of $39 \%$ risks poor representation of the sample population. However, this response rate is higher than previously published surveys in this field $[30,31]$ and literature supports this proportion as being adequate for generalisability.[38, 39] However, as we were interested in the teams' assessment and management of delirium, and several of the responding APM members might be part of the same team, this survey may not have obtained a geographically representative response. This could not be mitigated as many respondents work across settings and in order to maintain anonymity we could neither access specific team data nor identify individual teams. We did not include questions about practices in relation to prevention of delirium.

The responses to some of the questions are subjective and provisions of numerical estimates are likely to be approximate. Free-text comments indicated a lack of clarity for "refractory delirium" and "palliative sedation". Open online surveys are subject to selection bias due to a volunteer effect.[40] In this survey, an overrepresentation of motivated respondents with experience and interest in delirium might have skewed the results. This survey was principally answered by experienced palliative medicine physicians and the results have limited generalizability outside of this group. 


\section{Further research}

The findings from this survey emphasise the need for several other research areas. These include assessing clinicians' management of people with mild-moderate delirium and management of severe delirium in imminently dying patients, and the impact of research on clinical practice, and the implementation of existing evidence into practice. A key area is determining what would make clinicians change their practice when new evidence emerges. Further research is needed to understand physician decision-making processes when presented with delirium in a clinical context and how the care environment (community or hospice or hospital) impacts this decision-making.

\section{CONCLUSION}

This survey of UK Palliative Medicine specialists shows that delirium screening at inpatient admission is suboptimal. Systematic delirium screening should be completed using a tool and patient/family consultation on admission and on an ongoing basis. Most specialists continue to use antipsychotics in combination with non-pharmacological interventions to manage delirium. More structured support for family carers, backed up by palliative care specific information leaflets, should be routinely provided by clinical teams. Further rigorously designed clinical trials, especially to identify the standard of care for people with severe delirium, are urgently needed in view of management variability, emerging evidence and perceived priorities for research. There is also an urgent need to integrate findings from recent research into clinical practice. 


\section{Statements.}

\section{Contributorship:}

JWB, JS and PGL conceptualised, planned and constructed the study. JWB and MK led on data collection and analysis. All authors were involved in data interpretation. JWB and MK drafted the manuscript. All authors edited the manuscript and approved the final version.

Funding: No funding was received for this study.

Competing Interests: None of the authors have any Competing Interests to declare.

\section{Exclusive Licence:}

The Corresponding Author has the right to grant on behalf of all authors and does grant on behalf of all authors, an exclusive licence (or non-exclusive for government employees) on a worldwide basis to the BMJ Publishing Group Ltd and its Licensees to permit this article (if accepted) to be published in BMJ Supportive \& Palliative Care editions and any other BMJPGL products to exploit all subsidiary rights, as set out in our licence.

(http://group.bmj.com/products/journals/instructions-for-authors/licence-forms/).

Ethics statement: Hull York Medical School (UK) Research Ethics approval was attained prior to survey distribution.

Acknowledgments: The authors would like to thank everyone who took part in the survey. 


\section{References:}

1. American Psychiatric Association. Diagnostic and Statistical Manual of Mental Disorders, Fifth Edition (DSM-5), 5th edition: American Psychiatric Association, Arlington, 2013.

2. Lawlor PG, Bush SH. Delirium in patients with cancer: assessment, impact, mechanisms and management. Nat Rev Clin Oncol 2015;12(2):77-92. doi: 10.1038/nrclinonc.2014.147

3. de la Cruz M, Ransing V, Yennu S et al. The frequency, characteristics, and outcomes among cancer patients with delirium admitted to an acute palliative care unit. Oncologist 2015;20(12):1425-1431. doi: 10.1634/theoncologist.2015-0115

4. Hosie A, Davidson PM, Agar M et al. Delirium prevalence, incidence, and implications for screening in specialist palliative care inpatient settings: A systematic review. Palliat Med 2013;27(6):486-498. doi: 10.1177/0269216312457214

5. Pendlebury S, Lovett N, Smith SC et al. Observational, longitudinal study of delirium in consecutive unselected acute medical admissions: age-specific rates and associated factors, mortality and re-admission. BMJ Open 2015;5(11):e007808. doi: 10.1136/bmjopen-2015-007808

6. Salluh JIF, Wang H, Schneider EB et al. Outcome of delirium in critically ill patients: systematic review and meta-analysis. BMJ 2015;350:h2538. doi: 10.1136/bmj.h2538

7. Dani M, Owen LH, Jackson TA et al. Delirium, Frailty, and Mortality: Interactions in a prospective study of hospitalized older people. J Gerontol A Biol Sci Med Sci 2018;73(3):415-418. doi: 10.1093/gerona/glx214

8. Finucane AM, Lugton J, Kennedy C, Spiller JA. The experiences of caregivers of patients with delirium, and their role in its management in palliative care settings: an integrative literature review. Psychooncology 2017;26(3):291-300. doi: 10.1002/pon.4140

9. Carbone MK, Gugliucci MR. Delirium and the family caregiver: The need for evidence-based education interventions. Gerontologist 2015;55(3):345-352. doi: 10.1093/geront/gnu035

10. Toye C, Matthews A, Hill A, Maher S. Experiences, understandings and support needs of family carers of older patients with delirium: a descriptive mixed methods study in a hospital delirium unit. Int J Older People Nurs 2014;9(3):200-208. doi: 10.1111/opn.12019

11. de la Cruz M, Fan J, Yennu S et al. The frequency of missed delirium in patients referred to palliative care in a comprehensive cancer center. Support Care Cancer 2015;23(8):24272433. doi: 10.1007/s00520-015-2610-3

12. Inouye SK, Westendorp RG, Saczynski JS. Delirium in elderly people. Lancet 2014;383(9920):911-922. doi: 10.1016/S0140-6736(13)60688-1

13. Hosie A, Agar M, Lobb E et al. Palliative care nurses' recognition and assessment of patients with delirium symptoms: A qualitative study using critical incident technique. Int J Nurs Stud 2014;51(10):1353-1365. doi: 10.1016/j.ijnurstu.2014.02.005

14. Elsayem AF, Bruera E, Valentine AD et al. Delirium frequency among advanced cancer patients presenting to an emergency department: A prospective, randomized, observational study. Cancer 2016;122(18):2918-2924. doi: 10.1002/cncr.30133

15. Leonard MM, Agar M, Spiller JA et al. Delirium diagnostic and classification challenges in palliative care: subsyndromal delirium, comorbid delirium-dementia, and psychomotor subtypes. J Pain Symptom Manage 2014;48(2):199-214. 
16. Hshieh TT, Yue J, Oh E et al. Effectiveness of multicomponent nonpharmacological delirium interventions: a meta-analysis. JAMA Intern Med 2015;175(4):512-520. doi:

10.1001/jamainternmed.2014.7779

17. Leonard MM, Nekolaichuk C, Meagher DJ et al. Practical assessment of delirium in palliative care. J Pain Symptom Manage 2014;48(2):176-190.

18. Inouye SK, Bogardus ST, Jr., Charpentier P et al. A multicomponent intervention to prevent delirium in hospitalized older patients. N Engl J Med 1999;340(9):669-676.

19. Neufeld KJ, Yue J, Robinson TN et al. Antipsychotic medication for prevention and treatment of delirium in hospitalized adults: A systematic review and meta-analysis. $J \mathrm{Am}$ Geriatr Soc 2016;64(4):705-714. doi: 10.1111/jgs.14076

20. Schrijver EJM, De Graaf K, De Vries OJ et al. Efficacy and safety of haloperidol for inhospital delirium prevention and treatment: A systematic review of current evidence. Eur J Int Med 2016;27:14-23. doi: 10.1016/j.ejim.2015.10.012

21. Breitbart W, Marotta R, Platt M et al. A double-blind trial of haloperidol, chlorpromazine, and lorazepam in the treatment of delirium in hospitalized AIDS patients. Am J Psychiatry 1996;153(2):231-237. doi: 10.1176/ajp.153.2.231

22. Breitbart W, Alici Y. Evidence-based treatment of delirium in patients with cancer. J Clin Oncol 2012;30(11):1206-1214. doi: 10.1200/JCO.2011.39.8784

23. Agar MR, Lawlor PG, Quinn S. Efficacy of oral risperidone, haloperidol, or placebo for symptoms of delirium among patients in palliative care: A randomized clinical trial. JAMA Intern Med 2017;177(1):34-42. doi: 10.1001/jamainternmed.2016.7491

24. Hui D, Frisbee-Hume S, Wilson A et al. Effect of lorazepam with haloperidol vs haloperidol alone on agitated delirium in patients with advanced cancer receiving palliative care: A randomized clinical trial. JAMA 2017;318(11):1047-1056. doi: 10.1001/jama.2017.11468

25. Candy B, Jackson KC, Jones L et al. Drug therapy for delirium in terminally ill adult patients. Cochrane Database Syst Rev 2012;11:CD004770. doi: 10.1002/14651858.CD004770.pub2

26. Bush SH, Tierney S, Lawlor PG. Clinical assessment and management of delirium in the palliative care setting. Drugs 2017;77(15):1623-1643. doi: 10.1007/s40265-017-0804-3

27. National Clinical Guideline Centre. Delirium: diagnosis, prevention and management. Clinical Guideline 103. National Clinical Guideline Centre (NCGC) - Acute and Chronic Conditions, 2010. Available at: http://www.nice.org.uk/CG103

28. Morandi A, Davis D, Taylor JK et al. Consensus and variations in opinions on delirium care: a survey of European delirium specialists. Int Psychogeriatr 2013;25(12):2067-2075. doi: $10.1017 /$ S1041610213001415

29. Canadian Coalition for Seniors' Mental Health. Guideline on the assessment and treatment of delirium in older adults at the end of life: Canadian Coalition for Seniors' Mental Health (CCSMH), Toronto, 2010. Available at: https://ccsmh.ca/projects/delirium/

30. Lowe E, Elliot V, Owen R et al. How do UK palliative care physicians manage delirium? Results of an online survey. European Journal of Palliative Care 2016;23(3):110-113.

31. Agar M, Currow DC, Plummer J et al. Differing management of people with advanced cancer and delirium by four sub-specialties. Palliat Med 2008;22(5):633-640. doi: $10.1177 / 0269216308088691$

32. Claessens P, Menten J, Schotsmans P, Broeckaert B. Palliative Sedation: A review of the research literature. J Pain Symptom Manage 2008;36(3):310-333. doi: 10.1016/j.jpainsymman.2007.10.004 
33. Seymour J, Rietjens J, Bruinsma S et al. Using continuous sedation until death for cancer patients: A qualitative interview study of physicians' and nurses' practice in three European countries. Palliat Med 2015;29(1):48-59. doi: 10.1177/0269216314543319

34. Maltoni M, Scarpi E, Rosati M et al. Palliative sedation in end-of-life care and survival: a systematic review. J Clin Oncol 2012;30(12):1378-1383. doi: 10.1200/JCO.2011.37.3795

35. Bush SH, Lawlor PG, Ryan K, et al. Delirium in adult cancer patients: ESMO Clinical Practice Guidelines. Ann Oncol 2018;29(Supplement_4):iv143-iv65. doi: 10.1093/annonc/mdy147

36. Tropea J, Slee J, Holmes ACN et al. Use of antipsychotic medications for the management of delirium: an audit of current practice in the acute care setting. Int Psychogeriatr 2009;21(1):172-179. doi: 10.1017/S1041610208008028

37. Day J, Higgins I. Adult family member experiences during an older loved one's delirium: a narrative literature review. J Clin Nurs 2015;24(11-12):1447-1456. doi: 10.1111/jocn. 12771

38. Dobrow M, Orchard M, Golden B et al. Response audit of an internet survey of health care providers and administrators: implications for determination of response rates. $J$ Med Internet Res 2008;10(4): $\mathrm{e} 30$.

39. Johnson T, Wislar J. Response rates and nonresponse errors in surveys. JAMA 2012;307(17):1805-1806.

40. Eysenbach G, Wyatt J. Using the Internet for surveys and health research. J Med Internet Res 2002;4(2):E13. doi: 10.2196/jmir.4.2.e13 